\title{
WestVirginiaUniversity
}

THE RESEARCH REPOSITORY @ WVU

Graduate Theses, Dissertations, and Problem Reports

2016

\section{Chebyshev Polynomials and Fibonacci Numbers}

Nurul Wahyuni

Follow this and additional works at: https://researchrepository.wvu.edu/etd

\section{Recommended Citation}

Wahyuni, Nurul, "Chebyshev Polynomials and Fibonacci Numbers" (2016). Graduate Theses, Dissertations, and Problem Reports. 6885.

https://researchrepository.wvu.edu/etd/6885

This Thesis is protected by copyright and/or related rights. It has been brought to you by the The Research Repository @ WVU with permission from the rights-holder(s). You are free to use this Thesis in any way that is permitted by the copyright and related rights legislation that applies to your use. For other uses you must obtain permission from the rights-holder(s) directly, unless additional rights are indicated by a Creative Commons license in the record and/ or on the work itself. This Thesis has been accepted for inclusion in WVU Graduate Theses, Dissertations, and Problem Reports collection by an authorized administrator of The Research Repository @ WVU. For more information, please contact researchrepository@mail.wvu.edu. 


\title{
Chebyshev Polynomials and Fibonacci Numbers
}

\author{
Nurul Wahyuni \\ Thesis submitted \\ to the Eberly College of Arts and Sciences \\ at West Virginia University \\ in partial fulfillment of the requirements for the degree of \\ Master of Science in \\ Mathematics
}

Dr. Kevin Milans., Chair
Dr. Harvey Diamond
Dr. Michael Mays
Department of Mathematics

\begin{abstract}
Department of Mathematics
\end{abstract}
Morgantown, West Virginia 2016

Keywords: chebyshev polynomials, fibonacci numbers, combinatorics

Copyright 2016 Nurul Wahyuni 


\section{ABSTRACT \\ Chebyshev Polynomials and Fibonacci Numbers \\ Nurul Wahyuni}

The Chebyshev polynomials arise in several mathematical contexts such as approximation theory, numerical integration, and differential equations. Here we study a combinatorial interpretation of Chebyshev polynomials due to Shapiro, and we use it to give a slight variation of a combinatorial proof of Binet's Formula due to Benjamin, Derks and Quinn. Another beautiful formula for the Fibonacci numbers involves complex roots of unity. Presently, no combinatorial proof is known. We give combinatorial proofs of some related identities as progress toward a full combinatorial proof. 


\section{Contents}

1 Introduction 1

2 Combinatorial Interpretation of Chebyshev Polynomials 3

2.1 Chebyshev Polynomials of the Second Kind . . . . . . . . 3

2.2 Chebyshev Polynomials of the First Kind . . . . . . . . . . 5

2.3 Chebyshev and Trigonometry . . . . . . . . . . . 6

3 Another Proof for Binet's Formula 9

4 Fibonacci and Roots of Unity 12

4.1 Current Incomplete Work . . . . . . . . . . . . . . . . . 12

4.1.1 Tiling Approach . . . . . . . . . . . . . . . . 13

4.1.2 Graph Theoretic Approach . . . . . . . . . . 17

5 Conclusion $\quad 22$

$\begin{array}{lr}\text { Bibliography } & 23\end{array}$ 


\section{Chapter 1}

\section{Introduction}

Definition 1 The Chebyshev polynomials of the first kind are defined by the recurrence relation $T_{0}(x)=1, T_{1}(x)=x$, and for $n \geq 2$,

$$
T_{n}(x)=2 x T_{n-1}(x)-T_{n-2}(x) .
$$

Definition 2 The Chebyshev polynomials of the second kind are defined by the recurrence relation $U_{0}(x)=1, U_{1}(x)=2 x$, and for $n \geq 2$,

$$
U_{n}(x)=2 x U_{n-1}(x)-U_{n-2}(x) .
$$

Here is a list of the first few terms of Chebyshev polynomials of the first and second kind

Table 1.1: First few terms for each sequence

\begin{tabular}{lll}
$n$ & $T_{n}$ & $U_{n}$ \\
\hline 0 & 1 & 1 \\
1 & $x$ & $2 x$ \\
2 & $2 x^{2}-1$ & $4 x^{2}-1$ \\
3 & $4 x^{3}-3 x$ & $8 x^{3}-4 x$ \\
4 & $8 x^{4}-8 x^{2}+1$ & $16 x^{4}-12 x^{2}+1$
\end{tabular}

At first sight, there is nothing special with these polynomials. However, when we pick some special values for $x$, these polynomials become so special. For instance, when $x=i / 2$, where $i \in \mathbb{C}$ and $i^{2}=-1$, Chebyshev polynomials of the second kind are related to Fibonacci numbers, $U_{n}(i / 2)=i^{n} f_{n}$, 
where $f_{n}=F_{n+1}$, the shifted Fibonacci numbers. When $x=\cos \theta$, we obtain nice trigonometric identites $T_{n}(\cos \theta)=\cos (n \theta)$ and $U_{n}(\cos \theta)=$ $\sin ((n+1) \theta) / \sin \theta$. 


\section{Chapter 2}

\section{Combinatorial Interpretation of Chebyshev Polynomials}

First of all, let us recall the combinatorial interpretation for Fibonacci numbers. Traditionally, Fibonacci numbers are defined by $F_{0}=0, F_{1}=1$ and $F_{n}=F_{n-1}+F_{n-2}$ for $n \geq 2$. But in combinatorics, it is more natural to define $f_{n}=F_{n+1}$. The shifted Fibonacci $f_{n}$ counts the ways to tile a strip of length $n$ with squares and dominoes (see [1]). For example, $f_{3}=3$ and $f_{4}=5$ count 3 tilings of length 3 and 5 tilings of length 4 below:
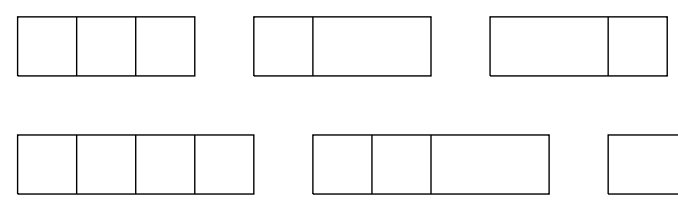

It turns out that Chebyshev polynomials count the same object but with a weight assigned to each tile.

Definition 3 In a tiling whose tiles are assigned weights, the weight of the tiling is the product of the weights of its tiles.

\subsection{Chebyshev Polynomials of the Second Kind}

Due to its simpler interpretation, we begin with Chebyshev polynomials of the second kind. $U_{0}=1, U_{1}=2 x$, and $U_{n}=2 x U_{n-1}-U_{n-1}$ for $n \geq 2$ has an interpretation as follow (see [2]). 
Theorem 4 For $n \geq 0, U_{n}(x)$ is the total weight of weighted tilings of length $n$ when squares have weight $2 x$ and dominoes have weight -1 .

Proof. We proceed by induction. For $n=0$, empty tiling has weight 1 . For $n=1$, we can only put a square tile, and the weight is $2 x$. So the initial conditions agree with the recurrence. Since any tiling begins with either a square or a domino, we can partition the tilings of length $n \geq 2$ into two groups, the tilings whose initial tile is a square and the tilings whose initial tile is domino. Since a square has weigh $2 x$ and followed by $(n-1)$-tiling which has total weight $U_{n-1}(x)$, the first group has total weight $2 x U_{n-1}(x)$. Since a domino has weight -1 and followed by $(n-2)$-tiling which has total weight $U_{n-2}(x)$, the second group has total weight $(-1) U_{n-2}(x)$.

\begin{tabular}{|l|l|l|l|}
\hline $2 x$ & $U_{n-1}(x)$ \\
\hline
\end{tabular}$\quad$ or \begin{tabular}{|l|l|}
\hline-1 & $U_{n-2}(x)$ \\
\hline
\end{tabular}

Hence $U_{n}=2 x U_{n-1}+(-1) U_{n-2}=2 x U_{n-1}-U_{n-2}$.

Here we provide the tilings of length two, three, four which sum to Chebyshev polynomials $U_{2}(x), U_{3}(x), U_{4}(x)$.

\begin{tabular}{|l|l|l|}
\hline $2 x$ & $2 x$ & -1 \\
\hline
\end{tabular}

\begin{tabular}{|l|l|l|l|l|l|l|l|}
\hline $2 x$ & $2 x$ & $2 x$ & $2 x$ & -1
\end{tabular}$\quad$\begin{tabular}{ll|l|}
\hline-1 & $2 x$ \\
\hline
\end{tabular}

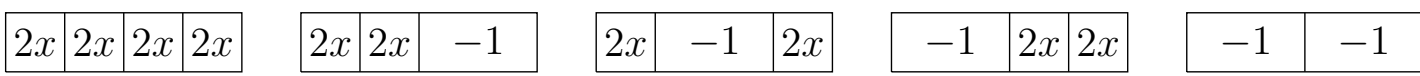

We can see that the total weight of the first row is $4 x^{2}-2=U_{2}(x)$, and for the second row $8 x^{3}-2 x-2 x=8 x^{3}-4 x=U_{3}(x)$ and the last one has total weight $16 x^{4}-4 x^{2}-4 x^{2}-4 x^{2}+1=16 x^{4}-12 x^{2}+1=U_{4}(x)$.

We can extend this model into a colored tiling interpretation by splitting the square into two squares of different colors. But now since we have two kind of squares, each square has weight half of the beginning, which is $x$. So, by giving the squares a color, black and white, we will have a colored interpretation as follow (see [2]).

Corollary 5 For $n \geq 0, U_{n}(x)$ counts the sum of the weights of $n$-tilings with dominoes, white squares and black squares, by giving all dominoes a weight of -1 , all white squares and black squares a weight of $x$. 


\subsection{Chebyshev Polynomials of the First Kind}

Chebyshev polynomials of the first kind only differ in their initial condition. Due to their initial condition in which $T_{1}(x)=x$, we have a restriction for the model. To make a model which agree with $T_{1}(x)=x$, and the recurrence still follow the same rules, it is not surprising that we just need to make en exception at it's initial square, the square which is located at the first cell. So here is the interpretation for Chebyshev polynomials for the first kind (see $[2])$.

Theorem 6 For $n \geq 0, T_{n}(x)$ is the total weight of weighted tilings of length $n$ where an initial square has weight $x$, all other squares have weight $2 x$, and all dominoes have weight -1 . We will call this a restricted tiling.

Proof. We use induction. It is easy to verify that the first two terms are good. Now consider a configuration of length $n \geq 2$. Due to it's restriction at the initial tile, we cannot calculate the total weight by conditioning at the first tile. Instead, we look at the last tile. We partition the tilings into two groups, the first group consisting all tilings ended with a square and the second group consisting all tilings ended with a domino. Since a square which is not an initial has weight $2 x$ and the first $(n-1)$-tiling has total weight $T_{n-1}(x)$, the first group has total weight $2 x T_{n-1}(x)$. By the same logic, the second group has total weight $(-1) T_{n-2}(x)$.

\begin{tabular}{|l|l|l|l|}
\hline$T_{n-1}(x)$ & $2 x$ \\
\hline
\end{tabular} or \begin{tabular}{|l|l|}
\hline$T_{n-2}(x)$ & -1 \\
\hline
\end{tabular}

Hence $T_{n}(x)=2 x T_{n-1}(x)-T_{n-2}(x)$.

Here we provide all configurations for $T_{2}(x), T_{3}(x)$, and $T_{4}(x)$.

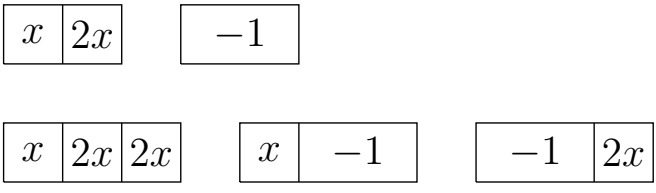

\begin{tabular}{|l|l|l|l|l|l|l|l|l|l|l|l|l|l|l|l|}
\hline$x$ & $2 x$ & $2 x$ & $2 x$ \\
\hline$x$ & $2 x$ & -1 \\
\hline
\end{tabular}$\quad$\begin{tabular}{lll}
$x$ & -1 & $2 x$ \\
\hline
\end{tabular}$\quad$\begin{tabular}{|l|l|l|l|}
\hline-1 & $2 x$ & $2 x$ \\
\hline
\end{tabular}

Here we can see that the total weight of the first row is $2 x^{2}-1=T_{2}(x)$, the second rows has total weight $4 x^{3}-x-2 x=4 x^{3}-3 x=T_{3}(x)$ and the third rows has total weight $8 x^{4}-2 x^{2}-2 x^{2}-4 x^{2}+1=8 x^{4}-8 x^{2}+1=T_{4} x$. 
For the colored tiling model, since $T_{n}(x)$ has a restriction that the initial square has weight half of the other squares, the initial square of any color has weight $x / 2$. Furthermore, another interpretation can be made by requiring an initial square to be white and assigning an initial square a weight of $x$. Hence, we have these two result (see [2]).

Corollary 7 For $n \geq 0, T_{n}(x)$ is the sum of the weights of $n$-tilings with dominoes, white squares and black squares by giving all dominoes a weight of -1 , all white squares and black squares a weight of $x$, except that the initial square has weight $x / 2$.

Corollary 8 For $n \geq 0, T_{n}(x)$ is the sum of the weights of $n$-tilings with dominoes, white squares and black squares by giving all dominoes a weight of -1 , all squares a weight of $x$ with the restriction that the initial tile must be either a white square or a domino.

The colored interpretation will help us in the next section to deal with trigonometric identities involving Chebyshev polynomials.

\subsection{Chebyshev and Trigonometry}

When we substitute $x=\cos \theta$ in each kind of Chebyshev polynomials, we obtain these two upcoming nice theorems. And it is even more amazing because we have combinatorial proof for them.

Recall De Moivre's theorem $e^{i n \theta}=\cos (n \theta)+i \sin (n \theta)$, which implies

$$
\cos (n \theta)=\frac{1}{2}\left(e^{i n \theta}+e^{-i n \theta}\right) \quad \text { and } \quad \sin (n \theta)=\frac{1}{2 i}\left(e^{i n \theta}-e^{-i n \theta}\right) .
$$

Since $\cos \theta=\frac{1}{2}\left(e^{i \theta}+e^{-i \theta}\right)$, we can interpret $T_{n}(\cos \theta)$ as the sum of the weight of colored tilings by assigning all white squares a weight of $e^{i \theta}$, all black squares a weight of $e^{-i \theta}$, and all dominoes a weight of -1 with restriction the initial white square has weight $\frac{1}{2} e^{i \theta}$ and initial black square has weight $\frac{1}{2} e^{-i \theta}$.

Since the second kind Chebyshev polynomials has no exception, $U_{n}(\cos \theta)$ is the sum of the weight of colored tilings by assigning all white squares a weight of $e^{i \theta}$, all white squares a weight of $e^{-i \theta}$, and all dominoes a weight of -1 . So now, we are ready to see combinatorial proof for these two theorems (see $[2])$. 
Theorem 9 For $n \geq 0$,

$$
T_{n}(\cos \theta)=\cos (n \theta)
$$

Proof. We need to show the sum of the weights of colored $n$-tilings with weights described above is equal to $\frac{1}{2}\left(e^{i n \theta}+e^{-i n \theta}\right)$. Notice that the $n$-tiling consisting all white squares has weight $\frac{1}{2} e^{i n \theta}$ and the one consisting all black squares has weight $\frac{1}{2} e^{-i n \theta}$. So to prove this identity we need to show the weights of the rest sum to zero.

In the other configurations, there exists the smallest index $j$ such that cells $j$ and $j+1$ are occupied by a domino or by squares of different colors. We consider two cases.

If $j>1$, we pair the tiling where cells $j$ and $j+1$ are occupied by a domino to the configuration where cells $j$ and $j+1$ are two squares of different colors and leave the rest unchanged. If the tiles before the domino are all white square then change the domino to white-black squares, otherwise change the domino to black-white squares. Since a domino has weight -1 and two different colors which is not at the beginning have weight $e^{i \theta} e^{-i \theta}=1$, this pair sums to zero.

For $j=1$, we pair each tiling initialing with a domino to the two tilings obatined by replacing the domino with an oppositely colored squares. The total weight sums to zero because the initial white-black contributes a weight of $\left(\frac{1}{2} e^{i \theta}\right) e^{-i \theta}=\frac{1}{2}$ and the initial black-white contributes a weight of $\left(\frac{1}{2} e^{-i \theta}\right) e^{i \theta}=\frac{1}{2}$, which sum to 1 .

Theorem 10 For $n \geq 0$,

$$
U_{n}(\cos (\theta))=\frac{\sin ((n+1) \theta)}{\sin \theta} .
$$

Proof. This is equivalent to $(\sin \theta) U_{n}(\cos (\theta))=\sin ((n+1) \theta)$. Since $\sin \theta=$ $\frac{1}{2 i}\left(e^{i \theta}-e^{-i \theta}\right)$, the left hand side of our theorem is

$$
\frac{e^{i \theta}}{2 i} U_{n}(\cos \theta)+\left(-\frac{e^{-i \theta}}{2 i}\right) U_{n}(\cos \theta) .
$$

Hence, the left hand side is the sum of the weights of $n+1$-tilings, where the first tile is a special white square with weight $\frac{e^{i \theta}}{2 i}$ or a special black square with weight $-\frac{e^{-i \theta}}{2 i}$ and then followed by $n$-tilings with no restriction, i.e. $U_{n}(\cos \theta)$. 
Meanwhile, the right hand side is

$$
\sin ((n+1) \theta)=\frac{1}{2 i}\left(e^{i(n+1) \theta}-e^{-i(n+1) \theta}\right) .
$$

The $n+1$-tiling consisting all white squares has weight $\frac{e^{i \theta}}{2 i} \cdot\left(e^{i \theta}\right)^{n}=\frac{1}{2 i} e^{i(n+1) \theta}$ and the one consisting all black squares has weight $-\frac{e^{-i \theta}}{2 i} \cdot\left(e^{-i \theta}\right)^{n}=-\frac{1}{2 i} e^{i(n+1) \theta}$.

Like what we did in the previous theorem, we will show the rest of the weights sum to zero. Again, let $j$ be the first cell such that cells $j$ and $j+1$ contain a domino or two squares of different colors. When $j>1$, the correspondence is the same as in Theorem 9 . When $j=1$, the initial blackwhite contributes a weight of $-\frac{e^{-i \theta}}{2 i} e^{i \theta}=-1 / 2 i$ and an initial white-black contributes a weight of $\frac{e^{i \theta}}{2 i} e^{-i \theta}=1 / 2 i$. Thus the weights the tilings which have the form black-white-X and white-black-X sum to zero. 


\section{Chapter 3}

\section{Another Proof for Binet's Formula}

Recall Binet's formula for Fibonacci numbers:

$$
F_{n}=\frac{1}{\sqrt{5}}\left[\left(\frac{1+\sqrt{5}}{2}\right)^{n}-\left(\frac{1-\sqrt{5}}{2}\right)^{n}\right] .
$$

Binet's formula was first established using generating functions by Abraham De Moivre in 1718 and then independently rediscovered by Jasques Binet (1843) and Gabriel Lamé (1844). The first combinatorial proof for this was using probability (see [1], [3], [6]). A more elegant combinatorial proof was then presented in [4] by creating a weighted tiling interpretation for Fibonacci numbers.

Here we will see a combinatorial proof for this formula using a Chebyshev polynomial identity of the second kind. This simple identity that we found in [9] is going to be useful.

\section{Theorem 11}

$$
U_{n}(i / 2)=i^{n} f_{n}
$$

Proof. $U_{n}(i / 2)$ is interpreted as the sum of weights of $n$-tilings of dominoes and squares where each domino has weight -1 and each square have weight $i$. Since $-1=i \cdot i$, by viewing the value of every domino as $i \cdot i$, any configuration of length $n$ have weight $i^{n}$. 


\section{Theorem 12}

$$
U_{n}(i / 2)=i^{n} \frac{1}{\sqrt{5}}\left[\left(\frac{1+\sqrt{5}}{2}\right)^{n+1}-\left(\frac{1-\sqrt{5}}{2}\right)^{n+1}\right]
$$

Proof. It is equivalent to proving

$$
(i \sqrt{5}) U_{n}(i / 2)=\left[\left(\frac{i+i \sqrt{5}}{2}\right)^{n+1}-\left(\frac{i-i \sqrt{5}}{2}\right)^{n+1}\right] .
$$

Let $\alpha=\frac{i+i \sqrt{5}}{2}$ and $\beta=\frac{i-i \sqrt{5}}{2}$. Observe some nice facts that $\alpha+\beta=i$ and $\alpha-\beta=i \sqrt{5}$, and moreover $\alpha \beta=1$. Hence the left hand side of our identity is $(\alpha-\beta) U_{n}\left(\frac{1}{2}(\alpha+\beta)\right)$ and the right hand side is $\alpha^{n+1}-\beta^{n+1}$. For our combinatorial purpose, we will distribute the left hand side to be

$$
(\alpha) U_{n}((\alpha+\beta) / 2)+(-\beta) U_{n}((\alpha+\beta) / 2) .
$$

Using the colored tiling interpretation, $U_{n}((\alpha+\beta) / 2)$ is the sum of weighted tilings with dominoes, white squares, and black squares by giving all dominoes a weight of -1 , all white squares a weight of $\alpha$ and all black squares a weight of $\beta$. Hence 3.1 can be interpreted as the sum of weights of all $(n+1)$-tilings with the restrictions the initial tile must be a square and a black initial square has weight $-\beta$.

On the right hand side, pick any $n+1$-tiling which initial tiling is a square. The tiling consists all white squares has weight $\alpha^{n+1}$ and the tiling with all black squares is going to be $-\beta^{n+1}$ since the first black square has weight $-\beta$. Now we will see that the sum of the rest configurations is zero.

Tilings other than those are tilings which involve dominoes or white and black squares together. In those cases, there exist a smallest index $j$ such that cells $j$ and $j+1$ are occupied by a domino or cells $j$ and $j+1$ are occupied by squares of different colors. Now let's consider two cases for where $j$ is located.

Case 1: $j>1$. If cells $j$ and $j+1$ are occupied by a domino, then pair this tiling with a tiling in which cells $j$ and $j+1$ are filled by different color of squares and the rest is the same. The decision of the color of squares depends on what is before the domino. It is either all black or all white. If it is all black then the domino is replaced by black white, otherwise white black, as 
illustrated below. In the other case, if cells $j$ and $j+1$ are occupied by squares of different colors, then replace them with a domino. Since domino has weight -1 and two different color which is not in the initial has weight $\alpha \beta=\beta \alpha=1$, we get a one-to-one correspondence which sum up to zero.

Case 2: If $j=1$, since the initial tiling is a square, the first two cells has weight $\alpha \beta$ in case it is white black or $(-\beta) \alpha$ in case it is black white. Put these into one-to-one correspondence by leaving the rest unchanged. Since $\alpha \beta+(-\beta) \alpha=0$, these will sum up to zero.

Corollary 13 Since $F_{n}=f_{n-1}$, theorem 1 and 2 will result in the $n^{\text {th }}$ Fibonacci formula that we have in the beginning.

This proof is actually a slight variation from the one in [4], in which they evaluate $U_{n}(x)$ at $x=1 / 2$ instead of $i / 2$. One can show that $-U_{n}(1 / 2)=f_{n}$. Then the proof proceeds similarly. 


\section{Chapter 4}

\section{Fibonacci and Roots of Unity}

\subsection{Current Incomplete Work}

Besides the famous Binet's formula, we found another way to express Fibonacci numbers explicitly (see [9]) as

$$
F_{n}=\prod_{k=1}^{\lfloor(n-1) / 2\rfloor}\left(1+4 \cos ^{2} \frac{k \pi}{n}\right)
$$

The algebraic proof for 4.1 can be found in [5]. Until now we have not seen any combinatorial proof for this. Since Binet's formula can be proved combinatorially, it is natural to ask for a combinatorial proof of 4.1, which is our main goal in this chapter. In this chapter, we present some related identities.

Recall that $2 \cos \theta=e^{i \theta}+e^{-i \theta}$. So,

$$
\begin{aligned}
F_{n} & =\prod_{k=1}^{\lfloor(n-1) / 2\rfloor}\left(1+4 \cos ^{2} \frac{k \pi}{n}\right) \\
& =\prod_{k=1}^{\lfloor(n-1) / 2\rfloor}\left(1+\left(e^{(k \pi i) / n}+e^{-(k \pi i) / n}\right)^{2}\right) \\
& =\prod_{k=1}^{\lfloor(n-1) / 2\rfloor}\left(3+e^{k(2 \pi i) / n}+e^{-k(2 \pi i) / n}\right) .
\end{aligned}
$$


For our convinience, we can write

$$
F_{n}=\prod_{k=1}^{\lfloor(n-1) / 2\rfloor}\left(3+\zeta^{k}+\zeta^{-k}\right)
$$

where $\zeta=e^{(2 \pi i) / n}$, a primitive $n$th root of unity.

\subsubsection{Tiling Approach}

Our approach on finding the combinatorial interpretation of 4.1 is by studying the combinatorial properties of this polynomial

$$
A_{n}(x) \stackrel{\text { def }}{=} \prod_{k=0}^{n-1}\left(x+\zeta^{k}+\zeta^{-k}\right)
$$

where $\zeta=e^{(2 \pi i) / n}$. Our aim is to see the combinatorial interpretation when $x=3$. But, here are the special cases for $x=0$ and $x=2$ which are also interesting.

Theorem 14 For $n \geq 1$ and $\zeta=e^{(2 \pi i) / n}$,

$$
A_{n}(0)=\prod_{k=0}^{n-1}\left(\zeta^{k}+\zeta^{-k}\right)= \begin{cases}2, & \text { if } n \equiv 1 \quad \text { or } 3 \quad(\bmod 4) \\ 0, & \text { if } n \equiv 0 \quad(\bmod 4) \\ -4, & \text { if } n \equiv 2 \quad(\bmod 4)\end{cases}
$$

Proof. By expanding $\prod_{k=0}^{n-1}\left(\zeta^{k}+\zeta^{-k}\right), A_{n}(0)$ can be interpreted as the total weight of weighted tilings of a strip of length $n$ in which the square at position $i$ has weight either $\zeta^{i}$ or $\zeta^{-i}$ for $0 \leq i \leq n-1$.

Define $W_{a, b}$ as the set of tilings $t$ having $a$ cells with positive power of $\zeta$ and $b$ cells with negative power of $\zeta$ and for $t \in W_{a, b}, w(t)$ denotes the weight of tiling $t$. For example, this tiling $t$

$$
\begin{array}{|l|l|l|l|l|}
\hline \zeta^{0} & \zeta^{-1} & \zeta^{2} & \zeta^{-3} & \zeta^{-4} \\
\hline
\end{array}
$$

belongs to $W_{2,3}$ since it has 2 positive powers and 3 negative powers and $w(t)=\zeta^{-6} . W_{a, b}$ is closed under cyclic sign rotation. Rotating the sign of a tiling in $W_{a, b}$ of weight $\alpha$ produce a tiling of weight $\zeta^{a} \zeta^{-b} \alpha$. To see how this 
works, multiply each $\zeta$ of positive power by $\zeta^{1}$ and each $\zeta$ of negative power by $\zeta^{-1}$. For instance, let $w(t)=\zeta^{0} \zeta^{1} \zeta^{-2} \zeta^{3} \zeta^{-4} \in W_{3,2}$, then $\zeta^{3} \zeta^{-2} w(t)=$ $\zeta^{-0} \zeta^{1} \zeta^{2} \zeta^{-3} \zeta^{4} \in W_{3,2}$. Now we will see how this cyclic pattern helps us in grouping the tilings that vanish in the summation.

Case 1: $n$ is odd. So now we have

$$
\zeta^{a-b} \sum_{t \in W_{a, b}} w(t)=\sum_{t \in W_{a, b}} w(t) \quad \text { which implies }\left(\zeta^{a-b}-1\right) \sum_{t \in W_{a, b}} w(t)=0 .
$$

Note that $\zeta^{a-b}=1$ if and only if $n \mid a-b$. If $a \geq 1$ and $b \geq 1$, since $a$ and $b$ are at most $n, n \mid a-b$ if and only if $a-b=0$ or $a=b$. But since $a+b=n$ and $n$ is odd, this will not happen. So, when $a \geq 1$ and $b \geq 1$, we have $\zeta^{a-b} \neq 1$, which then implies $\sum_{\alpha \in W_{a, b}}=0$. The only contributions for the sum are when $(a, b)=(n, 0)$ and $(a, b)=(0, n)$. In the first case we have

$$
\zeta^{0} \zeta^{1} \cdots \zeta^{n-1}=\zeta^{n(n-1) / 2}=\left(\zeta^{n}\right)^{(n-1) / 2}=1
$$

and in the second case we have

$$
\zeta^{-0} \zeta^{-1} \cdots \zeta^{-(n-1)}=\zeta^{-n(n-1) / 2}=\left(\zeta^{-n}\right)^{(n-1) / 2}=1
$$

since $(n-1) / 2$ is an integer. So we have $A_{n}(0)=2$ for odd integer $n$.

Case $2: n \equiv 0(\bmod 4)$. Since $n \equiv 0(\bmod 4), n / 4$ is an integer, moreover $\zeta^{n / 4}=i$ and $\zeta^{-n / 4}=-i$. Pair every tiling with $\zeta^{n / 4}$ at the $n / 4$ position with the tiling having $\zeta^{-n / 4}$ at the $n / 4$ position and leaving the rest unchanged. Since $\zeta^{n / 4}$ and $\zeta^{-n / 4}$ has the same magnitude and opposite sign, every pair sums to zero. Therefore $A_{n}(0)=0$ when $n \equiv 0(\bmod 4)$.

Case $3: n \equiv 2(\bmod 4)$. We split every tiling into two tilings, the odd indices and the even indices. Note that our index here start from 0 to $n-1$. Now in each group we have odd number of cells. We will work on one group, and fix the other group.

Consider the even indexes configuration. Let $\alpha$ be a configration in $W_{a, b}$. Note that now $a$ is how many positive powers of $\zeta$ in even index cells and $b$ is how many negative powers of $\zeta$ in even index cells. So the configuration in the odd indexes can be anything but it is fixed. Instead of multiplying with $\zeta^{a} \zeta^{-b}$, we now multiply with $\zeta^{2 a} \zeta^{-2 b}$ because to shift the signs in even indexes we need to move 2 cells ahead. Hence we have,

$$
\zeta^{2(a-b)} \sum_{t \in W_{a, b}} w(t)=\sum_{t \in W_{a, b}} w(t) \quad \text { which implies }\left(\zeta^{2(a-b)}-1\right) \sum_{t \in W_{a, b}} w(t)=0 .
$$


$\zeta^{2(a-b)}=1$ if and only if $n \mid 2(a-b)$ or $\frac{n}{2} \mid a-b$. Note that $a+b=n / 2$ and $n / 2$ is an odd integer. Using the similar argument as in case 1 , this is not the case when $a \geq 1$ and $b \geq 1$. Therefore when $a \geq 1$ and $b \geq 1, \sum_{t \in W_{a, b}} w(t)=0$. Similarly, when working on odd index cells, we have $\sum_{\alpha \in W_{a, b}}=0$ when $a \geq 1$ and $b \geq 1$. Hence the only contributions are when in each group we have all the powers of $\zeta$ with the same sign. There are 4 possibilities,

- Both groups have all positive powers

$$
\zeta^{0} \zeta^{1} \cdots \zeta^{n-1}=\zeta^{n(n-1) / 2}=\left(\zeta^{n / 2}\right)^{n-1}=(-1)^{n-1}=-1
$$

since $n-1$ is odd.

- Both groups have all negative powers

$$
\zeta^{-0} \zeta^{-1} \cdots \zeta^{-(n-1)}=\zeta^{-n(n-1) / 2}=\left(\zeta^{-n / 2}\right)^{n-1}=(-1)^{n-1}=-1
$$

since $n-1$ is odd.

- Even index cells have all positive powers and odd index cells have all negative powers

$$
\begin{aligned}
\left(\zeta^{0} \zeta^{2} \cdots \zeta^{n-2}\right)\left(\zeta^{-1} \zeta^{-3} \cdots \zeta^{-(n-1)}\right) & =\left(\zeta^{n(n-2) / 4}\right)\left(\zeta^{-n^{2} / 4}\right) \\
& =\left(\zeta^{n / 2}\right)^{(n-2) / 2}\left(\zeta^{-n / 2}\right)^{n / 2} \\
& =(-1)^{(n-2) / 2}(-1)^{n / 2} \\
& =1 \cdot-1=-1
\end{aligned}
$$

since $(n-2) / 2$ is even and $n / 2$ is odd.

- Even index cells have all negative powers and odd index cells have all positive powers

$$
\begin{aligned}
\left(\zeta^{-0} \zeta^{-2} \cdots \zeta^{-(n-2)}\right)\left(\zeta^{1} \zeta^{3} \cdots \zeta^{(n-1)}\right) & =\left(\zeta^{-n(n-2) / 4}\right)\left(\zeta^{n^{2} / 4}\right) \\
& =\left(\zeta^{-n / 2}\right)^{(n-2) / 2}\left(\zeta^{n / 2}\right)^{n / 2} \\
& =(-1)^{(n-2) / 2}(-1)^{n / 2} \\
& =1 \cdot-1=-1
\end{aligned}
$$

since $(n-2) / 2$ is even and $n / 2$ is odd. 
Since each configuration contributes -1 , we have $A_{n}(0)=-4$ when $n \equiv 2$ $(\bmod 4)$.

Theorem 15 For $n \geq 1$ and $\zeta=e^{(2 \pi i) / n}$,

$$
A_{n}(2)=\prod_{k=0}^{n-1}\left(2+\zeta^{k}+\zeta^{-k}\right)= \begin{cases}0, & \text { if } n \text { is even } \\ 4, & \text { if } n \text { is odd }\end{cases}
$$

Proof. $A_{n}(2)$ can be interpreted as the total weight of weighted tilings of a strip of length $n$ tiled with only squares where squares at position $i$ has weight either 2 or $\zeta^{i}$ or $\zeta^{-i}$ for $0 \leq i \leq n-1$.

Case 1: $n$ is even. We can simply pair each tiling with weight 2 at the $n / 2$-position with two tilings we obtain by replacing 2 with $\zeta^{n / 2}=-1$ and $\zeta^{-n / 2}=-1$ and leave the rest unchanged. These three tilings have total sum zero.

Case 2: $n$ is odd. Note that $2+\zeta^{k}+\zeta^{-k}=1+\zeta^{k}+\zeta^{-k}+\zeta^{k} \zeta^{-k}=$ $\left(1+\zeta^{k}\right)\left(1+\zeta^{-k}\right)$.

So,

$$
\prod_{k=0}^{n-1}\left(2+\zeta^{k}+\zeta^{-k}\right)=\prod_{k=0}^{n-1}\left(1+\zeta^{k}\right) \prod_{k=0}^{n-1}\left(1+\zeta^{-k}\right) .
$$

So, we can make another interpretation of $A_{n}(2)$ as the total weight of the weighted tilings of $2 \times n$-rectangle tiled with squares of different weights. For the first row, the weight of the square at position $i$ is either 1 or $\zeta^{i}$, and for the second row, the weight of the square at position $i$ is either 1 of $\zeta^{-i}$, where $0 \leq i \leq n-1$. Let $a$ be the number of $\zeta$ appear on the first row, and $b$ the number of $\zeta$ appear in the second row so $0 \leq a, b \leq n$. We will work on one row and fix the other row.

Define $W_{a, b}$ as usual, so if $t \in W_{a, b}$, then $t$ has $a$ cells with positive power of $\zeta$ in the first row and $b$ cells with a negative power of $\zeta$ in the second row. For example this tiling below belongs to $W_{2,3}$.

\begin{tabular}{|c|c|c|c|c|}
\hline 1 & 1 & 1 & $\zeta^{3}$ & $\zeta^{4}$ \\
\hline 1 & $\zeta^{-1}$ & 1 & $\zeta^{-3}$ & $\zeta^{-4}$ \\
\hline
\end{tabular}

If $1 \leq a \leq n-1, \zeta^{a} \neq 1$. With the same logic as in the proof of previous theorem, by multiplying $\zeta^{a}$ to the first row, we have

$$
\zeta^{a} \sum_{t \in W_{a, b}} w(t)=\sum_{t \in W_{a, b}} w(t) \Rightarrow\left(\zeta^{a}-1\right) \sum_{t \in W_{a, b}} w(t)=0 \Rightarrow \sum_{t \in W_{a, b}} w(t)=0 .
$$


Now let $1 \leq b \leq n-1$, and fix any configuration on the first row. By multiplying $\zeta^{-b}$ to the second row, we have

$$
\zeta^{-b} \sum_{t \in W_{a, b}} w(t)=\sum_{t \in W_{a, b}} w(t) \Rightarrow\left(\zeta^{-b}-1\right) \sum_{t \in W_{a, b}} w(t)=0 \Rightarrow \sum_{t \in W_{a, b}} w(t)=0
$$

since $\zeta^{-b} \neq 1$. Hence the only contributions for the sum are when $(a, b) \in$ $\{(0,0),(0, n),(n, 0),(n, n)\}$. By calculating it we have the weight for each case 1 . Therefore $A_{n}(2)=4$ when $n$ is odd.

\subsubsection{Graph Theoretic Approach}

\section{Graph Interpretation of Matrix and Its Determinant}

A matrix $\left(a_{i j}\right)$ can be described as a weighted directed graph $G$ in which $a_{i j}$ is the weight of the edge $u_{j}$ to $u_{i}$. For example

$$
A=\left[\begin{array}{lll}
1 & 3 & 2 \\
1 & 2 & 3 \\
2 & 3 & 1
\end{array}\right]
$$

is interpreted as this graph below.

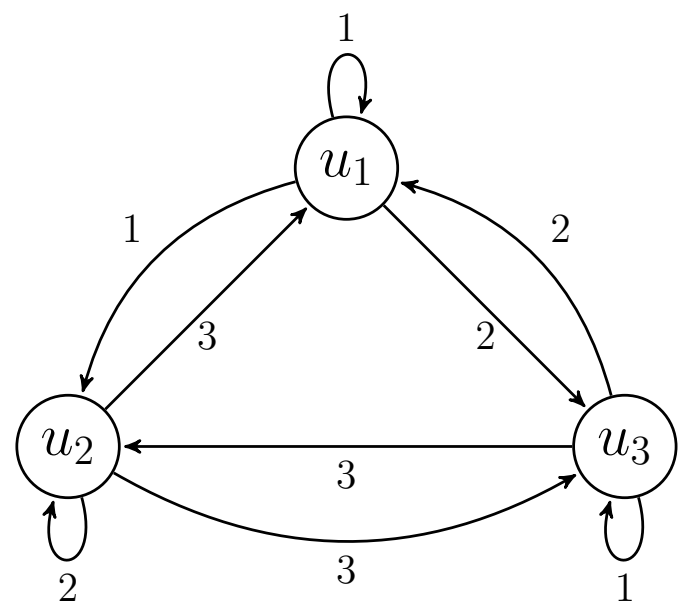

Definition 16 The weight of a weighted graph $G$ is the product of the weights of its edges.

In our example, the weight of graph $A$ is 216 . 
Definition 17 A 1-regular subgraph of a directed graph $G$ is a subgraph $F$ such that every vertex in $F$ has an in degree and out degree one.

The computation of the determinant of a matrix can be done in its graph representation using this theorem (see [7]).

Theorem 18 Let $R(G)$ be the set of all 1-regular subgraph of graph $G$. The determinant of a weighted directed graph $G$ is

$$
\sum_{F \in R(G)}(-1)^{\alpha(F)} w(F)
$$

where $\alpha(F)$ is the number of even cycles in $F$.

Let $B_{n}$ be an $n \times n$ matrix, with $n \geq 3$, such that the first row is $\left[\begin{array}{llll}x & 1 & 0 & 0\end{array}\right]$, and each subsequent row is obtained by cyclically shifting the entries of the previous row by one. So the second row of $B_{n}$ is $\left[\begin{array}{lllllll}1 & x & 1 & 0 & 0 & \ldots & 0\end{array}\right]$, and

$$
B_{n}=\left[\begin{array}{cccccc}
x & 1 & 0 & 0 & \ldots & 1 \\
1 & x & 1 & 0 & \ldots & 0 \\
0 & 1 & x & 1 & \ldots & 0 \\
\ldots & & & & & \\
1 & 0 & 0 & 0 & \ldots & x
\end{array}\right] .
$$

Theorem 19 For $n \geq 3$,

$$
\operatorname{det} B_{n}=A_{n}(x)=\prod_{k=0}^{n-1}\left(x+\zeta^{k}+\zeta^{-k}\right)
$$

Proof. We present an algebraic proof of this. Note that if $\lambda$ is an eigenvalue of $B_{n}$ with associated eigenvector $v$ then $A v=\lambda v$. The proof is by showing that $\left(x+\zeta^{k}+\zeta^{-k}\right)$ is an eigenvalue of $B_{n}$ with associated eigenvector 
$\left[1 \zeta^{k}\left(\zeta^{k}\right)^{2} \ldots\left(\zeta^{k}\right)^{n-1}\right]$. By matrix multiplication, for $0 \leq k \leq(n-1)$,

$$
\begin{aligned}
{\left[\begin{array}{cccccc}
x & 1 & 0 & 0 & \ldots & 1 \\
1 & x & 1 & 0 & \ldots & 0 \\
0 & 1 & x & 1 & \ldots & 0 \\
\vdots & & & & & \\
1 & 0 & 0 & 0 & \ldots & x
\end{array}\right]\left[\begin{array}{c}
1 \\
\zeta^{k} \\
\left(\zeta^{k}\right)^{2} \\
\vdots \\
\left(\zeta^{k}\right)^{n-1}
\end{array}\right] } & =\left[\begin{array}{c}
x+\zeta^{k}+\zeta^{-k} \\
1+x \zeta^{k}+\zeta^{2 k} \\
\zeta^{k}+x \zeta^{2 k}+\zeta^{3 k} \\
\vdots \\
1+\zeta^{-2 k}+x \zeta^{-k}
\end{array}\right] \\
& =\left[\begin{array}{c}
x+\zeta^{k}+\zeta^{-k} \\
\zeta^{k}\left(x+\zeta^{k}+\zeta^{-k}\right) \\
\zeta^{2 k}\left(x+\zeta^{k}+\zeta^{-k}\right) \\
\vdots \\
\left(\zeta^{n-1}\right)^{k}\left(x+\zeta^{k}+\zeta^{-k}\right) \\
1 \\
\\
\zeta^{k} \\
\left(\zeta^{k}\right)^{2} \\
\vdots \\
\left(\zeta^{k}\right)^{n-1}
\end{array}\right] .
\end{aligned}
$$

Hence $B_{n} v=\lambda v$. We claim that these eigenvectors are linearly independent. To see this, we can create a Vandermonde matrix $V$ by putting every eigenvector in a row of $V$. The determinant of this square Vandermonde matrix $V$ is

$$
\prod_{0 \leq i<j \leq n-1}\left(\zeta^{j}-\zeta^{i}\right) .
$$

Since all $\zeta^{i}$ are distinct, this number is nonzero. This implies $V$ has linearly independent rows. Therefore we obtain all of the eigenvalues of $B_{n}$. As always, the determinant of a matrix is the product of its eigenvalues. Hence we complete the proof.

Let us consider when $x=0$. The graph representation of $B_{n}$ will be a doubly oriented circle as shown below:

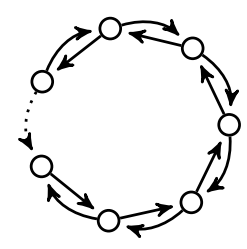


Note that all the edges in the graph have weight 1 . When $n$ is odd, the only 1-regular subgraphs are the long cycles $L_{1}$ and $L_{2}$. Since each of the long cycles has $\operatorname{sign}(-1)^{0}=1$, the determinant of $B_{n}$ is $2 \cdot 1=2$.

When $n$ is even we also have 1-regular subgraphs $F_{1}$ and $F_{2}$ in which every cycle has length 2 . The sign for $F_{1}$ and $F_{2}$ is $(-1)^{n / 2}$. Since $L_{1}$ and $L_{2}$ have a single cycle of even length, the sign for $L_{1}$ and $L_{2}$ is $(-1)^{1}=-1$. So the determinant is $2(-1)+2 \cdot(-1)^{n / 2}$. When $n \equiv 0(\bmod 4), n / 2$ is even, and therefore $2(-1)+2(-1)^{n / 2}=0$. When $n \equiv 2(\bmod 4), n / 2$ is odd, and therefore $2(-1)+2(-1)^{n / 2}=-4$. This is exactly what we have in Theorem 14.

Here is the general case.

Theorem 20 For $n \geq 3$,

$$
A_{n}(x)=2(-1)^{n+1}+\sum_{k=0}^{\lfloor n / 2\rfloor}(-1)^{k} \frac{n}{n-k}\left(\begin{array}{c}
n-k \\
k
\end{array}\right) x^{n-2 k}
$$

Proof. $A_{n}(x)$ is the determinant of matrix $B_{n}$ which is interpreted as the graph below.

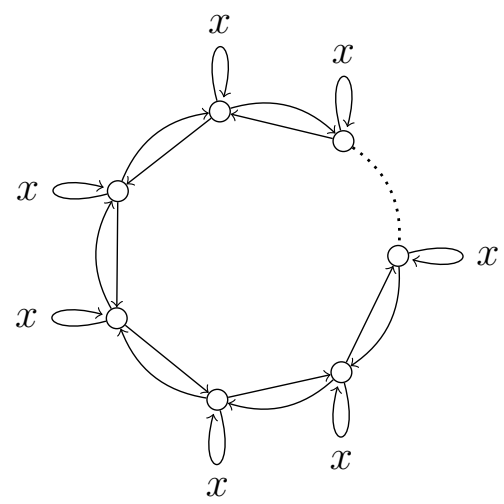

In this graph, every non loop edge has weight 1 .

There are 2 types of 1-regular subgraph here, long cycles and 2-cycles with some loops. There are 2 long cycles, one in each direction. If the numbers 
of vertices, $n$, is odd then the sign is $(-1)^{0}=1$, and if $n$ is even, the sign is $(-1)^{1}=-1$. So we can say the contribution from long cycles is $2(-1)^{n+1}$. Now, let's consider a 1-regular subgraph which consists of 2-cycles and/or loops. Every 1-regular subgraph $F$ with $k$ 2-cycles and $n-2 k$ loops will contribute to the coefficient of $x^{n-2 k}$. So the task is now to count the 1regular subgraphs having $k$ 2-cycles since the left over will just be the loops. Notice that it is equivalent to counting the matchings of size $k$ in the $n$-cycle $C_{n}$. The correspondence is by transforming every edge in the matching into a 2-cycle and vice versa. Note that the number of matchings of size $k$ in $C_{n}$ is (see [8])

$$
\frac{n}{n-k}\left(\begin{array}{c}
n-k \\
k
\end{array}\right) .
$$

For the subgraph $F$ with $k$ 2-cycles and $n-2 k$ loops, the sign will be $(-1)^{k}$ since every 2 -cycle is an even cycle and every loop is an odd cycle. The result follows by applying Theorem 19. 


\section{Chapter 5}

\section{Conclusion}

For the future research, one can continue trying to prove 4.1 combinatorially with our approach or using different methods. Specifically, let $\zeta=e^{(2 \pi i) / n}$.

- Since

$$
A_{n}(3)=\prod_{k=0}^{n-1}\left(3+\zeta^{k}+\zeta^{-k}\right)=5\left(\prod_{k=1}^{\lfloor(n-1) / 2\rfloor}\left(3+\zeta^{k}+\zeta^{-k}\right)\right)^{2},
$$

to complete the combinatorial proof of $F_{n}=\prod_{k=1}^{\lfloor(n-1) / 2\rfloor}\left(3+\zeta^{k}+\zeta^{-k}\right)$ one needs to show for $n \geq 3$,

$$
2(-1)^{n+1}+\sum_{k=0}^{\lfloor n / 2\rfloor}(-1)^{k} \frac{n}{n-k}\left(\begin{array}{c}
n-k \\
k
\end{array}\right) 3^{n-2 k}=5 F_{n}^{2} .
$$

- A combinatorial proof for

$$
A_{n}(x)=2(-1)^{n+1}+\sum_{k=0}^{\lfloor n / 2\rfloor}(-1)^{k} \frac{n}{n-k}\left(\begin{array}{c}
n-k \\
k
\end{array}\right) x^{n-2 k}
$$

is still an open problem. 


\section{Bibliography}

[1] A.T. Benjamin and J.J Quin. Proofs That Really Count: The Art of Combinatorial Proof. Mathematical Association of America, Washington, DC, 2003.

[2] A.T. Benjamin, L. Ericksen, P. Jayawant, and M. Shattuck. Combinatorial Trigonometry with Chebyshev Polynomials. Journal of Statistical Planning and Inference. 2010

[3] A.T. Benjamin, G.M. Levin, K. Mahlburg, and J.J Quinn. Random Approaches to Fibonacci Identities. The American Mathematical Monthly, Vol. 107, No. 6 (Jun.-Jul.,2000), 511-516.

[4] A.T. Benjamin, H. Derks, J.J. Quinn. The Combinatorialization of Linear Recurrences. Mathematics Subject Classifications, 05A19, $11 \mathrm{~B} 37$.

[5] B. Sury. Trigonometric Expressions for Fibonacci and Lucas Numbers. Acta Math. Univ. Comenianae. Vol. LXXIX, 2(2010), pp. 199-208.

[6] C.R.H. Hanusa, A.T. Benjamin, and F.E. Su. Linear Recurrences Through Tilings and Markov Chains.

[7] J.S. Maybee, D.D Olesky, P.V.D. Driessche, G. Wiener. Matrices, Digraph and Determinant. AMS Subject Classification 05C50, 15A15. 1987.

[8] L. Lovász, M.D. Plummer. Matching Theory. Amsterdam, Netherlands: North-Holland, 1986.

[9] N. Garnier, O. Ramaré. Fibonacci Numbers and Trigonometric Identities. AMS Classification: 11B39. 2006 